\title{
Linx
}

Revue des linguistes de l'université Paris X Nanterre

$61 \mid 2009$

Entre rection et incidence : des constructions verbales atypiques?

\section{Les incises en croire et cuidier en ancien français}

Julie Glikman

\section{OpenEdition}

Journals

Édition électronique

URL : http://journals.openedition.org/linx/1337

DOI : $10.4000 /$ linx.1337

ISSN : 2118-9692

Éditeur

Presses universitaires de Paris Nanterre

Édition imprimée

Date de publication : 1 juin 2009

Pagination : 71-85

ISSN : 0246-8743

Référence électronique

Julie Glikman, «Les incises en croire et cuidier en ancien français », Linx [En ligne], 61 | 2009, mis en ligne le 01 juin 2013, consulté le 01 mai 2019. URL : http://journals.openedition.org/linx/1337 ; DOI : 10.4000/linx.1337

Département de Sciences du langage, Université Paris Ouest 


\title{
Les incises en croire et cuidier en ancien français ${ }^{1}$
}

\author{
Julie Glikman \\ Lattice, ENS \& CNRS / \\ Modyco, Université Paris Ouest Nanterre \& CNRS
}

\section{Les incises de « recteurs faibles »}

\subsection{Définition}

La possibilité d'apparaître dans différentes constructions syntaxiques, suivis d'une proposition complétive (désormais abrégée que-P) ou en position incise, à la première personne du présent de l'indicatif, est définitoire des verbes que Urmson (1952) désigne par «verbes parenthétiques », et Blanche-Benveniste (1989) par « recteurs faibles » puis verbes « faibles » (Blanche-Benveniste $\&$ Willems 2007) (désormais abrégés RF) ${ }^{2}$ :

A verb which, in the first person present, can be used, as in the example above, followed by that and an indicative clause, or else can be inserted at the middle or end of the indicative sentence, is a parenthetical verb. (Urmson, 1952, p. 481)

\footnotetext{
1 Je tiens à remercier les participants de la journée d'étude « Entre rection et incidence » pour leurs commentaires, les relecteurs de la revue, ainsi que Sophie Prévost, Paola Pietrandrea et Nicolas Mazziotta pour leur aide et leurs conseils pour la finalisation de cet article.

${ }^{2}$ Voir introduction au volume, et les articles de Gachet et de Schneider (ici-même) pour un plus large aperçu des travaux sur la question.
} 
Je définirai la classe des «verbes recteurs faibles » par la double possibilité de construction qu'ils ont ; on peut les trouver en tête de la construction, suivis d'une que-phrase qui a les apparences d'un complément $[\ldots]$ ou en incise, après la séquence à apparence de complément (ou à l'intérieur de cette séquence). (BlancheBenveniste, 1989, p. 60)

La construction incise des RF est en outre définie par l'absence de lien syntaxique explicite entre celle-ci et l'énoncé hôte, et par l'absence de pronom objet, et donc l'absence de saturation de la valence du verbe incident, valence qui peut être récupérée sémantiquement dans l'hôte (Blanche-Benveniste 1989 ; Blanche-Benveniste \& Willems 2007 ; Schneider 200733). L'incise de RF est ainsi décrite comme réalisée en «je crois» et non en «je le crois» ou «comme je le crois». Cette dernière caractéristique distingue également, selon ces auteurs, l'incise de RF d'autres types de constructions incidentes avec reprise. À cette absence de pronom objet dans l'incise s'ajoute l'argument de l'impossibilité de reprise pronominale en le de la que-P ou de l'hôte, caractéristique également définitoire pour Blanche-Benveniste $(1989)^{4}$ : *il fait beau, je le crois. Ces caractéristiques et certaines restrictions d'emploi (voir BlancheBenveniste, 1989, pp. 65-66) ont parfois conduit certains chercheurs à assimiler le fonctionnement des RF au fonctionnement de certains adverbes modalisateurs du type heureusement (que), cette assimilation pouvant être perçue comme une analyse en termes de grammaticalisation des emplois des RF (ibid. p. 71 ; Andersen, 1996, p. 313). Selon Thompson \& Mulac (1991) la construction en incise $I$ think est à analyser, en diachronie, comme découlant de la construction rectrice $I$ think that $[\ldots]$.

\subsection{Problématique et hypothèse}

Cette possibilité d'apparaître à la première personne du présent dans différentes constructions, suivis qu'une que-P ou en construction incise, existe également pour un certain nombre de verbes en ancien français (désormais AF), notamment pour le verbe croire, verbe analysé comme RF en français moderne (désormais FM) ${ }^{5}$ :

(1) - Par foi, fait Lion, je croi bien que ce soit il, car il resemble mout bien mon seignor. (BFM, qgraal_cm, p. 297, p. 3)

Par foi, fait Lyon, je crois bien que ce soit lui, car il ressemble très bien à mon seigneur]

(2) car ceien₹, ne demorra il pas longuement ce sai ge bien por la grant queste dou Graal qui prochainnement comencera si com je croi et Lancelot le nos a bui fet entendant qu'il nel deïst pas s'il n'en seust aucune chose. (BFM, qgraal_cm, p. 297, p. 11)

\footnotetext{
${ }^{3}$ Schneider (2007) introduit ainsi pour les incises de RF la notion de « reduced parenthetical clause ».

${ }^{4}$ Cette idée d'impossibilité de reprise pronominale a cependant été modérée dans les études ultérieures (Blanche-Benveniste \& Willems 2007), qui reconnaissent la possibilité d'une reprise toujours non pas en le, mais en ce que.

${ }^{5}$ Ces différentes possibilités de constructions semblent exister pour de nombreux autres verbes en AF (voir Franzen 1939), ainsi qu'en FM (j'espère, je suppose...) mais pour un relevé exhaustif nous avons préféré limiter les possibilités lexicales, et prendre le verbe le plus prototypique dans les deux états de langue, croire.
} 
[car ici il ne demeurera pas longtemps, ce sais-je bien, pour la grande quête du Graal qui va commencer prochainement, comme je crois, et Lancelot nous l'a fait aujourd'hui comprendre $[\ldots]]$

(3) Ja n'i venra, ce croy, a point [...] (BFM, gcoin3, p. 344, v. 1054)

[il n'arrivera jamais, ce [régime] crois-je, à point]

Les constructions sont comparables, mais on observe dès le premier abord deux différences importantes avec les constructions incises telles que définies au point précédent: la présence d'un lien syntaxique avec l'hôte, "si com», en (2), et la présence d'un pronom régime, «ce», en (3). Dans ce contexte où l'absence de pronom et l'absence de lien syntaxique explicite dans l'incise sont données comme définitoire de la catégorie de RF, cette observation soulève un certain nombre de questions : la présence d'un pronom dans l'incise en AF est-elle un indice montrant que ces verbes n'ont pas un fonctionnement de RF dans cet état de langue? Ceci serait-il un argument pour dire que les incises de RF résulteraient d'une grammaticalisation, ou que les RF auraient connu un changement dans leur valence? Ou faut-il au contraire en conclure que c'est la définition des RF qui est à revoir?

Nous montrerons dans cet article qu'il ne s'agit pas d'un cas de grammaticalisation, et que les incises de l'AF constituent bien les mêmes types d'incises qu'en FM. Nous montrerons que les différences entre les deux états de langues sont à analyser comme une différence dans leur réalisation de surface, et que la présence du pronom est liée au type de données, écrites ou orales, et non à un changement syntaxique dans la construction ou la valence du verbe : bien que les incises de RF relève de l'oralité (Koch \& Oesterreicher 2001), ou d'une expression ou représentation de l'oralité (Marchello-Nizia à par.), leur forme de réalisation se fait en fonction du support, écrit ou oral. Nous commencerons pour cela par décrire le fonctionnement de ces verbes en AF (2.), puis nous interrogerons le rôle du pronom dans l'incise en AF (3.), et montrerons comment ce passage d'une incise en ce crois à une incise en je crois peut être analysé comme une différence de réalisation liée au type de données (4.).

\subsection{Méthodologie}

Pour étudier le comportement de ces verbes en AF, et voir les ressemblances et différences de comportement avec la catégorie des RF telle que définie pour le FM, nous avons procédé au relevé dans sept œuvres de la totalité des occurrences tous temps et toutes personnes confondus de croire ainsi que de cuidier (forme fréquente en $\mathrm{AF}$, quasi synonyme de croire et ayant le même type de comportement, totalement disparue par la suite au profit de croire et penser) :

- La Vie de Saint Alexis : vers, 11e siècle, vie de saint (abrégé Alexis);

- La Chanson de Roland: vers, 11e siècle, chanson de geste (abrégé Roland) ;

- Le Roman de Renart, branche X : vers, début 13e, parodie de chanson de geste (abrégé Renart);

- La conquête de Constantinople, Robert de Clari : prose, 13e, chronique historique (abrégé Clari);

- La Mort le roi Artu: prose, 13e, roman (abrégé Artu);

- Jean de Saintré, Antoine de La Sale : prose, 15e, roman (abrégé Saintré) ;

- La farce de Maistre Pathelin : vers, 15e, théâtre (abrégé Pathelin). 
À ce relevé systématique viennent s'ajouter des exemples de crois relevés dans Berte aus grans piés, Adenet le Roi (vers, 13e, chanson de geste, abrégé Berthe).

Nous avions au préalable inclus le verbe penser à cette étude, cependant, nous n'avons relevé dans notre corpus aucun exemple de constructions incises pour ce verbe $^{6}$. L'article étant centré sur la question du comportement de l'incise de RF en AF, nous avons donc, dans un deuxième temps, retiré de l'étude ce verbe, qui ne connait ainsi pas la construction incise en AF. Une recherche dans la base textuelle Frantext du moyen français et dans la Base de Français Médiéval (BFM) donne des occurrences de la construction incise en penser à partir du 14e siècle seulement : beau douce Sires et Meistres, et moustre a vous qe touz les sept mortels pecchés sont issuz, par ma bouche et tout pleyn ariere venuz en touz, ceo pense jeo ausi, par ceo malveis pertuz dequels j'ai charge a ma poure cheitive alme de rendre ent acounte, [...] (BFM, Henri de Lancastre, Le Livre de seyntz, medicines (1354) p. 15).

Pour l'étude du fonctionnement des constructions incises en AF, la définition d'incise adoptée doit être suffisamment large pour pouvoir prendre en compte les différentes configurations possibles existantes. Sont ainsi considérées comme incises les constructions verbales insérées à un point de la chaîne syntaxique d'un élément phrastique, appelé hôte, sans en changer la construction syntaxique. La position de l'incise peut être à l'intérieur de la chaine, ou en fin. Cependant, la question de la position finale peut créer en $\mathrm{AF}$ des cas d'ambiguité syntaxique entre construction rectrice et construction incise (voir Glikman 2010), nous avons ainsi écarté quelques constructions ambigües. Pour mieux cerner le phénomène de la construction incise dans son ensemble, nous avons également intégré au relevé les constructions introduites du type «comme je crois ».

\section{Premières observations}

\subsection{Les différentes constructions possibles}

De la même manière que pour le verbe croire en FM, notre corpus confirme que les verbes croire, cuidier ont la possibilité d'apparaittre dans différents schémas de constructions, régissant une que-P (celle-ci pouvant en AF être ou non introduite par $\left.q u e^{7}\right)$, ou en construction incise, à la première personne du présent de l'indicatif, ce comportement correspondant ainsi à la définition des "verbes parenthétiques » d'Urmson ou de « recteur faible » de Blanche-Benveniste :

(1) Si est blecét, ne quit que anme i remaigne. (Roland 1848)

[Ainsi il est blessé, je crois que l'âme n'y est plus] (que-P)

\footnotetext{
${ }^{6}$ Sur les cent soixante-et-unes occurrences du verbe relevées (hors emploi infinitif et nominal type «le penser »), nous avons trouvé dix-huit constructions employées de manière absolue («E Baligant cumencet a penser» (Roland 2789)), deux occurrences comme introducteur du discours direct (« et en ce faisant pensoit : «Las! et que ay je fait ? [...]»» (Saintré 7)), trente-neuf occurrences comme recteur de que-P et cent-une occurrence avec un régime nominal, et une seule occurrence pouvant être possiblement analysée comme une incise, mais non analysée comme telle du fait de l'ambiguiité d'analyse possible : « je atandrai ore le tans / que jel ferai, ce que je pans, / couroucié plus que ne fis onques. » (Renart 1922).

${ }^{7}$ Voir Glikman (2009) sur cette question.
} 
(2) Quïas le guant me caïst en la place, / Cum fist a tei le bastun devant Carle?» (Roland 764-65) [Crois-tu le gant me tombe des mains sur place, comme le bâton de Charles fit avec toi ?] ([que]-P)

(3) E si n avrez, co quid, de plus gentil: (Roland 150)

[Et vous en aurez, ce crois, de plus nobles] (incise)

Ils peuvent également être construits avec un régime nominal ${ }^{8}$ :

(4) Sire, jo vos en crei. (Roland 3463)

[Sire, je vous en crois] (nominal)

Dans les œuvres étudiées, sur les quatre cents onze occurrences relevées de nos verbes, la construction incise ne représente que vingt occurrences. La proportion d'incise par rapport au total des occurrences des verbes par texte n'est en outre pas la même, certains textes n'ayant aucune occurrence de construction incise : c'est le cas dans Clari, Alexis et Saintré. La répartition des différentes constructions dans les œuvres est la suivante ${ }^{9}$ :

\begin{tabular}{c|c|c|c|c|c|c|c|c} 
& Alexis & Roland & Renart & Clari & Artu & Saintré & Pathelin & Total \\
\hline Absolu & & & & & & & 1 & 1 \\
\hline Ambigu & & 1 & 1 & & & & 2 & 4 \\
\hline Incise & & 3 & 6 & & 5 & & 2 & 20 \\
\hline que-P & & 4 & 32 & 7 & 101 & 32 & 10 & 196 \\
\hline SN & 3 & 21 & 47 & 5 & 67 & 31 & 16 & 190 \\
\hline Total & 3 & 29 & 86 & 12 & 173 & 63 & 31 & 411
\end{tabular}

Nous reviendrons dans la partie suivante sur l'analyse qu'on peut faire de cette répartition.

\footnotetext{
${ }^{8}$ Cette possibilité de construction est également présente pour les verbes considérés comme « recteurs faibles » en FM, mais n'est que peu mise en relation avec ces autres constructions, parce qu'elle est alors considérée non plus comme une rection «faible », mais comme une rection " forte ", c'est-à-dire un emploi non parenthétique. Il nous semble cependant intéressant de tenir compte de ces constructions pour observer quelle part des occurrences du verbe l'emploi «faible» occupe. Nous avons regroupé par la suite dans les comptage comme régime nominal « $\mathrm{SN}$ » les constructions avec un régime nominal : Et se j'eüsse crë̈ Agravain vostre frere [...] (Artu 30), les constructions avec un régime pronominal «personnel» (reprenant une personne et non une proposition) : Seignors barons, jo vos aim, si vos crei (Roland 3410), ainsi que les constructions avec un régime infinitif (qui représentent un nombre important d'occurrences, mais qui n'entrent pas dans les contextes possibles de concurrence avec la construction incise, telle qu'on la définit habituellement pour les verbes RF, il ne nous a ainsi pas semblé nécessaire, à ce stade et pour le but de cet article, de les distinguer, mais ceci pourra faire l'objet d'une étude ultérieure) : il cuide parler a ses bestes (Pathelin 152). Dans le cas de Berthe, les chiffres donnés ne sont pas représentatifs, le relevé systématique de toutes les occurrences des verbes n'ayant pas été fait.

${ }^{9}$ Nous n'avons pas inclus au tableau Berthe, qui n'a pas fait l'objet d'un relevé systématique de toutes les occurrences des verbes.
} 


\subsection{La construction incise}

\subsubsection{Insertion dans l'hôte}

De la même manière qu'en FM, l'incise peut se trouver à différents endroits de la proposition hôte, insérée entre le verbe et son complément (5), ou dans une coordination de deux termes nominaux (6) :

(5) E si n avrez, ço quid, de plus gentil\%: (Roland 150) [et vous en aurez, ce crois, de plus nobles]

(6) Iert $i$ sis niés, li quens Rollant, ço crei, / E Oliver, li proz e li curteis. (Roland 575-76) [il y avait son neveu, le comte Roland, ce crois, et Olivier, le preux et le courtois]

On peut également la trouver en position finale, mais cette construction peut parfois provoquer une ambigüité entre construction rectrice en parataxe et construction incise :

(7) Dist Oliver: « sire cumpainz, ce crei, / De Sarrazins purum bataille aveir 》 (Roland 1006-07) [Olivier dit: «Sire compagnon, ce crois, nous pourrons avoir bataille avec les Sarrazins »]

(8) einz q'esisiez de la prison / $i$ eüstes tel livroison, / je cuit, tiex. c. cous $i$ eüstes, / que onques gré... (Renart 1733)

[avant que vous sortiez de la prison, vous avez eu un tel traitement, je crois, cent coups tels y avez eu,...]

\subsubsection{Les différents types d'incises}

Les incises peuvent apparaître dans notre corpus sous différents schémas de construction :

1) le type ce crois : avec pronom régime, sans pronom sujet (voir (5) et (6) cidessus) [tab. ce].

2) le type ce crois je : avec pronom régime et pronom sujet, ce type est visiblement plus tardif (deux exemples relevés dans Pathelin), et pourrait constituer le type intermédiaire entre « ce crois » et « je crois » (voir Féron 2005) ${ }^{10}$ [tab. ce;je] :

(9) ...la plus rebelle villenaille qui soit, se croy je, en ce royaume! (Pathelin 86)

[... la plus rebelle canaille qui soit, ce crois-je, en ce royaume !]

3) le type je crois : nous n'avons relevé qu'un exemple de ce type dans notre corpus, avec pronom sujet et sans pronom régime (comme la forme moderne), et qui en outre peut constituer un cas d'ambiguité [tab. non] :

\footnotetext{
${ }^{10}$ Pour penser, les bases textuelles donnent également des attestations de ce type à partir du 14e, Frantext donne par ex. : Et ainsi evesque seray, / Ce pense je; (anonyme --- Miracle de l'evesque que l'arcediacre murtrit, c.1341, 112) ; Nennil certes, je fais comme je doy : / Et se je tiens le party qu'ay choisy, /Riens n'ay meffait, ce pense je, vers toy. (Charles d'ORLÉANS, Ballades, c.1415-1457, 87).
} 
(10) Se ce ne fust, mon cors je croi eust honni. (Berthe)

[S’il n'avait pas été là, il aurait, je crois, déshonoré mon corps].

Franzen (1939 : 85) situe cependant les premières apparitions de « je crois » en remplacement de « ce crois » à la fin $12^{\mathrm{e}}$, début $13^{\mathrm{e}}$ siècle.

4) le type (si) comme je crois : construction incise sous la forme d'une subordonnée adverbiale, de manière générale la plus fréquente ${ }^{11}$ (voir tab. et aussi Schneider ici même), cette construction ne comporte pas de pronom régime, le comme étant dans cette construction uniquement conjonctif ${ }^{12}$ [tab. com] :

(11) Mais avant volez, mot a mot, / si con je croi, oïr trestot, [...] (Renart 3392)

[Mais avant vous voulez mot à mot, ainsi que je crois, entendre tout...]

Comme les autres types d'incises, ce type peut s'insérer à un endroit de la chaîne syntaxique de l'hôte sans en changer la construction (ici entre le modal vouloir et son complément infinitif ö̈r).

5) le type le crois : nous n'avons relevé qu'un exemple avec un pronom régime le, qui entre dans une construction « ainsi le crois », à rapprocher, nous semble-t-il, du type précédent, également introduit par un élément ouvrant la proposition ${ }^{13}$ [tab. le] :

(12) Fust morte, ainsi le croi, se ne fust rescaufee. (Berthe)

[Je serai morte, ainsi le crois, si je ne m’étais échappée.]

Ces différents types se répartissent comme suit dans les textes :

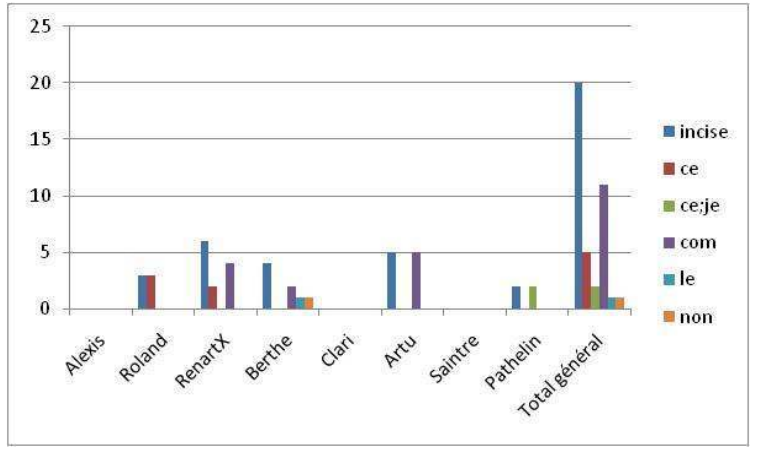

\footnotetext{
11 Possible également avec penser au 14e : Tu iés assés bien coustumiers / De faire un lay, sicom je pense. (Jean FROISSART, Le Paradis d'amour, c.1361-1362, 64, Frantext).

12 Nicolas Mazziotta me signale cependant qu'il est possible d'analyser comme et de lui donner une fonction à la fois de conjonction et à la fois de circonstant du verbe croire, analyse effectivement possible, mais qui dans tous les cas ne remplit pas la position régime, et comporte un marqueur de type adverbial en début de proposition.

13 Comme Nicolas Mazziotta me l'a également signalé, ainsi n'est pas forcément conjonctif ici, mais même s'il est analysé comme un circonstant de croire, on peut tout de même le considérer comme une présence d'un élément de type adverbial en tête de proposition.
} 
On peut regrouper ces types en deux ensembles différents. Les types 1) à 3) constituent l'incise de RF telle qu'elle est définie dans les descriptions des RF, sans lien syntaxique explicite avec l'hôte et à la première personne du présent de l'indicatif. La différence entre chacun de ces groupes, ainsi qu'avec les descriptions des incises de RF en FM, est la présence ou non du pronom régime ce et du pronom personnel sujet, ce dernier n'étant pas obligatoirement exprimé pendant toute la période de l'ancien français.

Le deuxième ensemble (type 4) et 5)) est celui des subordonnées adverbiales introduites. Comme nous l'avons déjà signalé, ces constructions ne sont habituellement pas traitées avec les autres, du fait de leur caractère introduit et, de ce fait, de leur statut syntaxique différent. Dans l'optique d'étudier le comportement de ces verbes en $\mathrm{AF}$, et de comprendre le comportement de la construction incise, il nous semble fondamental, à ce stade de l'étude de la diachronie des RF, de les inclure. Ce type de constructions incises comporte toujours le pronom sujet, et majoritairement pas de pronom régime : seul l'exemple de type 5) contient un pronom régime le, et, sur les onze occurrences du type «comme je crois», une seule présente un pronom régime, également de forme le: "Car il en est mout poi, ainsi com je le cuit»(Berthe). La construction en comme je crois (type 4), largement plus fréquente dans les textes, est aussi syntaxiquement plus libre. On peut la trouver à différents temps et à différentes personnes (trois exemples relevés) :

(13) mais il en garroit bien prochainement, si com il cuidoit. (Artu 116)

[mais il en guérirait bien prochainement, ainsi qu'il croyait.]

(14) ... que vos estes moult traveilliez, si com nos cuidons. Et li rois respont [...] (Artu 50)

[...que vous êtes très fatigué, ainsi que nous croyons. » Et le roi répond...]

Enfin, toutes les incises apparaissent dans du discours rapporté au style direct (DD), sauf deux cas du type comme je crois, qui apparaissent en narration (voir aussi (13) ci-dessus) :

(15) car il mouvra demain, si comme il cuide, a aler a la mer por passer en la terre de Gaunes. (Artu 120)

[car il partira demain, ainsi qu'il croit, pour aller à la mer pour passer en terre de Gaunes]

\section{Rôle du pronom dans l'incise de RF en AF et évolution}

\subsection{Sur la saturation du verbe par le pronom $C E$}

La présence du pronom régime ce ne vient pas saturer une valence obligatoire du verbe dans les incises de RF de l'AF. Le premier argument pour cette analyse est que le pronom ce peut être analysé en AF comme un pronom de reprise «faible », ne saturant pas nécessairement la valence verbale. Ceci est attesté par l'observation de ses autres emplois comme corrélatif annonçant une complétive qui, elle, sature la valence, ou, de la même manière que les incises de RF, sa présence dans les incises de DR (voir Glikman à par. et Mazziotta et al. 2012) : 
(16) Co sent Rollant que la mort le tresprent, (Roland 2354)

[Ce sent Roland que la mort le presse]

(17) Co dit li reis que sa guere out finee. (Roland 704)

[ce dit le roi qu'il a fini sa guerre]

(18) Icele tere, ço dit, dun il esteit, (Roland 979)

[cette terre, ce dit, dont il était...]

Ainsi, le pronom ce peut annoncer une que-P sans saturer la valence verbale, sorte de corrélatif. Pour Franzen, le ce vient ainsi «résumer» l'hôte, ce qui est effectivement le cas, cependant pas pour remplir une éventuelle nécessité de saturer la valence du RF, mais pour indiquer le lien entre les deux propositions, hôte et incise.

L'hypothèse d'une reprise "faible », dans le cas du pronom ce, est également confirmée par l'existence d'une reprise pronominale en le, correspondant à une reprise «forte », dans l'emploi « fort» du verbe :

(19) Vous dites, et je le croi bien, que par un chevalier sera li roiames de Logres desertés et tornés a destruction. (merlin_suite_litt, p.013, BFM)

[Vous dites, et je le crois bien, que le royaume de Logres sera détruit par un chevalier.]

Cette distinction entre une reprise « forte », en le, et une reprise « faible », en $c e$, rejoint ainsi l'analyse de Blanche-Benveniste \& Willems (2007), sur la distinction entre une reprise en ce que et une reprise en le, seule la première étant compatible avec un emploi «faible » des RF.

Un autre argument visant à montrer que le pronom ce ne vient pas saturer la valence du verbe tient au fait que dans les autres types d'incises, en "comme je crois », la valence du verbe n'est pas nécessairement saturée. Au contraire, comme nous l'avons vu, l'absence de pronom régime est largement majoritaire dans la construction en "comme je crois", (une occurrence avec pronom régime sur onze occurrences relevées). Dans le cas où le pronom régime est réalisé, il prend alors la forme le, et non $c e$, dans cette construction comme dans celle en « ainsi »14.

Ainsi, le pronom ce est à analyser comme un pronom de reprise «faible », et non «fort» comme le. Il ne vient pas nécessairement saturer la valence du verbe de l'incise. Dans les cas où l'incise est clairement délimitée et introduite par un marqueur de lien explicite, il n'est jamais réalisé. On peut en conclure que sa présence n'est plus nécessaire dans les cas où un autre type de marquage est réalisé, et qu'il joue, dans les cas où il est présent, ce même rôle de marqueur de lien syntaxique.

\footnotetext{
14 Cette possibilité de réalisation d'un pronom régime confirme en outre le fait que dans cette construction, le comme est uniquement conjonctif et ne vient pas saturer la valence du verbe.
} 


\section{2. Évolution de la construction incise}

Les incises en «ce crois » de l'AF se réalisent sous la forme « je crois » en FM, montrant ainsi un passage de «ce crois » à « je crois» en diachronie (Franzen, 1939, p. $97^{15}$ ). En latin, credo « je crois» pouvait également construire une incise de type parenthétique, construction apparemment suffisamment courante pour qu'elle soit mentionnée dans le Gaffiot. Les exemples cités dans Gaffiot proviennent des discours de Cicéron, dans lesquels credo semble fonctionner de la même manière que les incises parenthétiques décrites par Urmson (1952) ou Schneider (2007) :

(20) si te iam, Catilina, comprehendi, si interfici iussero, credo, erit verendum mibi ne non hoc potius omnes boni serius a me quam quisquam crudelius factum esse dicat. (M. Tullius Cicero, Against Catiline, Albert Curtis Clark Ed, site perseus)

Le pronom sujet pronominal n'étant pas obligatoire en latin (comme c'est toujours le cas en AF), il n'est pas présent dans l'incise, et on observe également qu'il n’y a pas, à la différence des constructions en « ce crois » de l'AF, de pronom régime.

$\mathrm{Au} 16^{\mathrm{e}}$ siècle $^{16}$, on observe encore les deux grands types d'incises, en « ce crois» et en «comme je crois». Le type en «comme je crois» représente vingtquatre occurrences, auxquelles s'ajoutent deux occurrences en « comme je le crois » :

(21) Mais ainsi Dieu soit à ton aide, comme je le croy! (LAVARDIN Jacques de La Celestine [adapt.], 1578, 55)

Sur les trente-deux occurrences d'incises de type "ce crois", comme on le voyait s'amorcer au $15^{\mathrm{e}}$ siècle avec le développement de l'expression obligatoire du sujet pronominal, on n'observe plus qu'un cas d'incise du type «ce crois» sans expression du pronom sujet, au début du siècle, les autres constructions apparaissant sous la forme « ce crois-je » :

(22) Le vela, ce croy! (Anonyme La Farce des théologastres, 1528, 103-104)

(23) Me voyci ce croy-je arrivé Devant l'huis d'Emee. (BAÏF Jean-Antoine Le Brave, 1573, 211)

Enfin, on relève sept occurrences en «je crois », sans pronom régime, toutes provenant de pièces de théatre :

\footnotetext{
${ }^{15}$ Franzen fait également remarquer qu'à l'inverse, il y a eu un passage de l'incise de discours rapporté en « ce dit » à « dit-il ». Selon lui, cette distinction tient notamment au fait de l'existence, en $\mathrm{AF}$, d'une variante de construction en «dit-il», majoritaire, qui a permis son maintien, tandis qu'il n'existait pas de variante en «crois-je ».

16 Pour le $16^{\mathrm{e}}$ siècle, nous avons recherché dans Frantext (période Renaissance : 1501-1600) les séquences «ce crois», «ce croy», « je crois » et «je croy» en excluant la présence de «que », puis nous avons vérifié manuellement les occurrences ainsi relevées.
} 
(24) Si brave guerrier que, je croy, Mars mesme, le dieu des combas, Avecque vous n'oseroit pas S'aparager - non sans raison -(BAÏF Jean-Antoine Le Brave, 1573, 48)

Ainsi, si la construction en «je crois » est déjà possible depuis la fin du $12^{\mathrm{e}}$ siècle d'après Franzen, les incises en «ce crois-je » sont encore largement plus fréquentes au $16^{\mathrm{e}}$ siècle, et présente jusqu’à la fin du siècle.

Lorsque l'on regarde en diachronie la construction de l'incise de RF de type « je crois ", on observe ainsi une évolution dans sa structure interne, depuis credo en latin jusqu'à je crois en FM :

$$
\begin{aligned}
& \text { latin } \mathrm{AF} \quad \mathrm{MF}-16^{\mathrm{e}} \quad \mathrm{FM} \\
& \text { credo }>\text { ce } \text { croi }>\text { ce crois } \text { je }>\text { je crois }
\end{aligned}
$$

Selon Franzen (1939, p. 116), l'introduction du pronom tient à des raisons prosodiques, la forme monosyllabique crois ou quis de l'AF étant dans l'incapacité de former à elle seule une proposition incise rythmiquement autonome, à la différence de la forme dissyllabique de première personne du présent du latin credo. Le développement de la réalisation du pronom sujet, d'abord dans un schéma " ce crois je », ordre normal en présence d'un objet avant le verbe, puis avec le développement de la tendance préférentielle à avoir le sujet placé avant le verbe, explique en partie, selon lui, le passage de « ce crois » à « je crois ». Cependant, l'explication prosodique ne suffit pas à expliquer le maintien aussi tardif du ce dans «ce crois-je » au $16^{\mathrm{e}}$ siècle, alors que la construction « je crois » est déjà présente, ni le fait qu'on ait préféré l'introduction du régime ce plutôt que d'un pronom sujet je qui aurait pu produire le même effet, du point de vue strictement prosodique. Il est à noter en outre qu'on n'observe aucune occurrence comprenant à la fois le pronom sujet réalisé avant le verbe et le pronom ce, ceci pouvant être analysé comme une preuve du caractère non nécessaire du pronom $c e$ en présence d'un autre élément marquant le début de la proposition.

\section{Une opposition oral/écrit: les différentes réalisations de la construction incise en fonction du type de données}

Les incises en «je/ce crois » sont attestées depuis longtemps, en ancien français, et en latin dans une construction de type $"$ credo $»^{17}$. Il ne s'agit ainsi pas d'une construction formée à partir de la construction «je crois que-P», comme le proposait Thompson \& Mulac (1991), tout du moins pas de formation récente. Ce type d'incises est au contraire, selon nous, à rapprocher des constructions incises en « comme je crois ». Le type en comme existe également dès l'ancien français, et semble se maintenir sous la même forme dans les différents états de langue. C'est en outre pour chaque période étudiée le type d'incises le plus fréquent (voir Schneider ici-même pour le 17 e siècle).

Notre analyse est que les incises en "ce crois» et les incises en «je crois» constituent bien le même type d'incise de RF. La différence entre les deux

\footnotetext{
17 Voir aussi Rochette (2007).
} 


\section{Julie Glikman}

constructions s'explique parfaitement à la lumière de la distinction opérée par Koch \& Oesterreicher (2001, voir aussi Koch 1995) entre la réalisation médiale (codes graphique et phonique) et le continuum conceptuel (immédiat communicatif (" oralité ») et distance communicative (" écrit»)). Dans les deux cas, il s'agit d'incises à la première personne du présent de l'indicatif, sans reprise pronominale forte, et relevant du pôle de l'oralité. On les trouve à l'oral pour les incises du FM, dans du discours rapporté au style direct (DD) en AF (sur les vingt occurrences, toutes apparaissent dans le DD, à l'exception de deux occurrences en comme). Les exemples latins proviennent également de discours de Cicéron destinés à être prononcés à l'oral, lors de plaidoyer ou de discours politiques, tandis qu'une recherche dans La guerre des Gaules de César ne donne aucun résultat de credo en construction incise. Cependant, elles ne relèvent pas du même médium (données orales ou données écrites). Cette différence de médium explique la différence de réalisation en fonction du type de données, le code phonique et le code graphique marquant différemment ce type d'incises. À l'écrit, le marquage doit revêtir une forme plus explicite, comme l'atteste le nombre bien plus important dans les données écrites des incises en « comme je crois ", où le lieur revêt une forme conjonctive $^{18}$. Le pronom régime ce dans les incises de RF de l'AF est ainsi un «lieur », servant au marquage de la construction incise dans des données écrites.

Cette prise en compte de l'opposition oral/écrit (code $v$ s conceptualisation) explique également la différence entre les incises de RF en « je crois» et les incises de DR en « dit-il ». En effet, l'apparition du pronom régime ce pourrait être rapprochée de l'opposition que montre Franzen entre «ce dit» et «dit-il». Franzen montre que le premier est principalement utilisé en discours rapporté au style indirect (exemple 25), alors que les incises en « dit-il » sont davantage employées dans le DD (Franzen, 1939, p. 86) :

(25) La vos sivrat, ço dit, mis avoę: (Roland 136)

Traduction de Bédier (citée par Franzen) : «Là vous suivra, il vous l'assure, mon seigneur.»

Pour Franzen, l'existence d'une construction en «dit-il», liée à un emploi particulier, explique la différence du passage de «ce crois» à «je crois», lorsque l'expression du sujet et l'ordre S - V ont été privilégiés. Les incises en «ce dit»sont passées à « dit-il », ou « qu'il dit» dans la «langue vulgaire » (Franzen, ibid., p. 97) du fait de l'existence de cette double possibilité de construction, tandis qu'il n'existait pas de construction en «crois-je » pouvant remplir ce rôle. Ceci ne suffit pas à expliquer la disparition de la construction «ce crois-je », mais la différence entre l'incise en «ce dit » et l'incise en « dit-il», telle que démontrée par Franzen, rejoint notre hypothèse d'une opposition oral-écrit, et offre ainsi une possibilité d'analyse qui pourrait être appliquée aux RF. En effet, pour Franzen, le ce est présent en DR indirect, mais nous pouvons voir que c'est lorsque un locuteur, au discours direct, rapporte les paroles d'autrui. Il s'agit donc de discours rapporté dans de l'oral représenté. Le choix du

18 La présence d'un marquage explicite, et donc fort, dans l'incise en «comme» explique leur caractère à la fois plus autonome (plus grande liberté de variation : la subordonnée adverbiale peut apparaittre à différentes personnes, temps, et c'est également le seul type à apparaître en passages narratifs (deux occurrences)) et plus stable. 
régime ce et non pas du sujet je tient alors à la nécessité de marquer l'insertion à l'hôte, insertion marquée par l'inversion dans certains cas de verbes de DR, ou par un marqueur plus explicite, comme, et ici par un pronom de reprise rappelant explicitement le lien avec l'hôte.

Zink (1997) explique le passage à «je crois» comme une mise en valeur du «moi » du locuteur, mais cette explication, tout en étant possiblement juste, nous semble devoir être complétée. L'augmentation des incises en «je crois», avec l'obligation de réalisation du sujet pronominal puis la préférence au sujet antéposé, le pronom je remplaçant ainsi le pronom ce, s'est également opérée parallèlement au développement de la ponctuation dans les textes, ou dans des cas permettant un autre type de marquage : en effet, les occurrences en « je crois » sont davantage attestées, au $16^{\mathrm{e}}$ siècle, dans des pièces de théâtre, destinées a priori à une performance orale, de même que le credo latin, tandis que les constructions en «ce crois je » sont très présentes chez Montaigne, plus proche du pôle écrit. De même, la présence plus importante des incises en comme se justifie de fait par le caractère écrit des données à disposition pour l'ancienne langue. En FM, les incises de RF sur lesquelles portent les études de Blanche-Benveniste (1989) et Blanche-Benveniste \& Willems (2007) proviennent toutes de données orales. La différence moderne entre «je crois» et « dit-il» peut ainsi s'expliquer par le fait que les incises de RF en « je crois » sont principalement étudiées en FM oral, alors que les incises en « dit-il » relèvent de l'écrit. Or, associer la présence du pronom régime $c e$ à un style particulier rejoint aussi le fait qu'on puisse avoir « je le crois » dans de l'oral « corrigé » et l'inversion du sujet dans les incises de RF dans de l'écrit en FM (voir Gachet même volume). Les deux types d'incises ne sont donc pas si différents, si on tient compte de la différenciation de marquage en fonction du type de données ${ }^{19}$.

\section{Conclusion}

La présence du pronom régime ce dans les incises en croire et cuidier de l'ancien français ne remet pas en question la définition de l'incise de recteur faible : ces incises peuvent ainsi être analysées comme des incises de recteur faible. Les verbes recteurs faibles étant défini par la possibilité d'avoir une construction incise de ce type, il est ainsi possible d'affirmer que croire connait dès l'ancien français (au moins) un fonctionnement de recteur faible. La différence de réalisation entre l'incise de RF du $\mathrm{FM}$, en je crois, et celle de l'AF, en ce crois, tient à la différence du type de données, oral ou écrit, mais leur présence dès l'AF montre qu'on peut étudier «l'oralité » dans des données écrites anciennes.

\footnotetext{
${ }^{19}$ Les incises de DD comme celles de RF indiquent un positionnement par rapport au discours hôte, une prise en charge ou non. On n'observe alors logiquement pas les mêmes personnes en fonction du but recherché et du type de verbe (il dit vs je crois).
} 


\section{RÉFÉRENCES BIBLIOGRAPHIQUES}

ANDERSEN, H. L. (1996), «Verbes parenthétiques comme marqueurs discursifs », in Muller C. (éd.), Dépendance et intégration syntaxique, Subordination, coordination, connexion, Tübingen, Niemeyer, pp. 307-315.

Blanche-Benveniste, C. (1989), «Constructions verbales 'en incise' et rection faible des verbes », Recherches sur le français parlé, $\mathrm{n}^{\circ} 9$, pp. 53-73.

Blanche-Benveniste, C. \& WiLlems, D. (2007) «Un nouveau regard sur les verbes faibles », Bulletin de la Société de linguistique de Paris, $\mathrm{n}^{\circ} 102 / 1$, pp. 217-254.

FÉRON, C. (2005), « Modalisation et verbes d'opinion : quelques remarques sur 'croire', 'cuidier' et 'penser' dans 'La Queste del Saint Graal' », L'information grammaticale, n¹04, pp. 15-21.

FRANZEN, S.R. (1939), Étude sur la syntaxe des pronoms personnels sujets en ancien français, Almqvist \& Wiksells Boktryckeri-A.-B.

GACHET, F. (ici-même), « Les verbes parenthétiques : un statut syntaxique atypique ?».

GAFFIOT, F. (1934), Dictionnaire Latin Français, Paris, Hachette.

GLIKMAN, J. (à par), «La double complémentation : double marquage ou dislocation ?», Actes du colloque ComplémentationS, Saint-Jacques de Compostelle, octobre 2010.

Glikman, J. (2009), Parataxe et subordination en ancien français. Système syntaxique, variantes, variation, Thèse de doctorat, Université Paris Ouest - Universität Potsdam.

Koch, P. \& Oesterreicher, W. (2001), «Langage parlé et langage écrit», Lexikon der Romanistichen Linguistik, t. I, Tübingen, Max Niemeyer Verlag, pp. 584-627.

KocH, P. (1995), "Subordination, intégration syntaxique et 'oralité' », La subordination dans les langues romanes, H. L. Andersen et G. Skytte (éds), Etudes Romanes 34, Copenhague.

MARCHELLO-NiziA, C. (à par.), «L' “oral représenté”, un accès construit à une face cachée des langues 'mortes'. Episodes de 'discours direct' en ancien français ", Actes du colloque Diachro V, Octobre 2010.

MAZZiotta N., Bischof B., GLikMAn J., RAinSFORD T. (2012), « Ce sujet dans les « constructions impersonnelles » du Roman de Tristan de Béroul », L'Information Grammaticale, n¹32, pp. $48-52$.

Rochette, B. (2007), «Les éléments hors stucture dans la Cena Trimalchionis. Remarques sur la structure de la phrase de Pétrone ", Elements "asyntaxiques" ou hors structure dans l'énoncé latin, C. Bodelot (éd.), Clermont Ferrand, Presses universitaires Blaise Pascal.

SCHNEIDER, S. (ici-même), «Les clauses parenthétiques dans des textes français du $17 \mathrm{e}$ et $18^{\mathrm{e}}$ siècle. Une analyse pragmatique, syntaxique et diachronique ».

Schneider, S. (2007), Reduced Parenthetical Clauses as Mitigators. A corpus study of spoken French, Italian and Spanish, Amsterdam, John Benjamins. 
Thompson, S. A. \& Mulac, A. (1991), «A Quantitative Perspective on the Grammaticization of Epistemic Parentheticals in English» in Traugott, E. \& Heine, B. (éds), Approaches to Grammaticalization, vol II, Focus on Types of Grammatical markers, Amsterdam / Philadelphia, pp. 313-329.

Urmson, J. O. (1952), « Parenthetical verbs », Mind, nº61 (244), pp. 480-496.

ZINK, G. (1997), Morphosyntaxe du pronom personnel (non réfléchi) en Moyen Français, Genève, Droz.

\section{CORPus}

Adenet le Roi, Berte aus grans piés, A. Henry éd., 1963, P U de Bruxelles, Bruxelles.

Antoine de la Sale, Jehan de Saintré, 1456, éd. J. Misrahi, C.A. Knudson, 1965, Genève, Droz.

Farce de Maistre Pierre Pathelin, 1456, éd. J. Dufournet, 1986, Paris, Garnier Flammarion.

La chanson de Roland, 1100, éd. G. Moignet, 1969, Paris, Bordas.

La Mort le roi Artu, 1230, éd. Frappier J., 1964, Genève, Droz.

La Vie de Saint Alexis, 1050, éd. Storey C., 1968, Genève, Droz.

Le roman de Renart (branche X), début 13e, éd. M. Roques, 1963, Paris, Champion.

Robert de Clari, La conquête de Constantinople, P. Lauer éd., 1924, Champion, Paris.

\section{Bases en ligne :}

- Perseus pour le latin : http://www.perseus.tufts.edu/hopper

- Frantext et Frantext médiéval pour les textes du 16e (Atilf) : http://www.frantext.fr/

- Base de Français Médiéval (ENS Lyon \& Icar) : http://bfm.ens-lyon.fr/ 